\title{
MASTERS: A Virtual Lab on Multimedia Systems for Telecommunications, Medical and Remote Sensing Applications
}

\author{
Dimitrios S. Alexiadis and Nikolaos Mitianoudis, Senior Member, IEEE,
}

\begin{abstract}
Digital Signal Processing (DSP) has been an integral part of most Electrical, Electronic and Computer Engineering curricula. The applications of DSP in multimedia (audio, image, video) storage, transmission and analysis are also widely taught at both the undergraduate and postgraduate levels, as digital multimedia can be encountered in most human daily activities. In this paper, a virtual lab on multimedia systems for telecommunications, medical and remote sensing applications is presented; consisting of 20 graphical user interfaces, it can support teaching activities for various DSP concepts. The virtual lab developed was used as a teaching companion for a postgraduate course on multimedia content management, to enhancing student comprehension of the material taught; it has been made freely available for similar educational or research purposes.
\end{abstract}

Index Terms-Demonstration, digital signal and image processing, interactive education, multimedia, visualization

\section{INTRODUCTION}

D IGITAL Signal Processing (DSP) describes a wide range of activities that can be performed for the efficient manipulation of signals (audio, static images, video and so on) in a discrete representation, using a personal computer or any other processing or embedded system [1]. Efficient DSP techniques have numerous applications in fields such as telecommunications, medicine, remote sensing and finance. Hence DSP, along with several derivative modules, including audio and image processing, has become an integral part of most Electrical, Electronic or Computer Engineering curricula worldwide [2]. Simultaneously, the advancement of several programming platforms, such as MATLAB, LabView, C++ and JAVA, has offered new powerful tools and libraries that can be used either for research or educational purposes [3], [4] in the field of DSP. The use of Graphical User Interfaces (GUI) to demonstrate the basic principles of DSP has been highlighted before, [3].

The School of Science and Technology ${ }^{1}$ at the International Hellenic University (IHU) was founded in 2010 and offers

Manuscript received January 30, 2012; revised xx xx. This project was funded by the Operational Program Education and Lifelong Learning, OPS 200056 (International Hellenic University, Thessaloniki, Greece).

Both authors were with the School of Science and Technology, International Hellenic University, Greece.

Dimitrios Alexiadis is currently with the Informatics and Telematics Institute, CERTH, Greece. (e-mail: dalexiad@iti.gr, tel: +30 2310 464160.)

Nikolaos Mitianoudis is currently with the Department of Electrical and Computer Engineering, Democritus University of Thrace, 67100 Xanthi, Greece (e-mail: nmitiano@ee.duth.gr, tel: +30 25410 79572, fax: +30 25410 79569.)

${ }^{1}$ http://www.tech.ihu.edu.gr/ two postgraduate programs, one being an M.Sc. in Information and Communication Technology (ICT) Systems. The Virtual Labs project prepared interactive supporting material for these programs, providing essential infrastructure for the Schools teaching and research activities. The virtual labs implement a number of GUI-based experiments on several key topics whose theory is covered in postgraduate-level modules. This paper presents the virtual laboratory (VLab) known as MASTERS (MultimediA Systems in Telecommunications, mEdical and Remote Sensing applications). The MASTERS VLab uses GUIs to implement several interactive exercises, giving students practical experience of important theoretical aspects of multimedia processing. The main objective of this VLab is to immerse the student in the related theoretical topics, starting with very fundamental concepts and progressively and hierarchically building up his/her knowledge to the level of current state-of-the-art approaches.

The MASTERS VLab is intended to be of use both to tutors giving in-class demonstrations and to students studying athome and performing laboratory practicals. In-class demonstrations using the interactive MASTERS GUIs can complement lectures, offering an immediate visual interpretation of the concepts discussed. The MASTERS VLab is accompanied by a detailed manual that provides an essential summary of the theoretical concepts behind each experiment, describes the structure and functionality of each GUI, and provides a set of unworked exercises which can be used as homework assignments. Since the MASTERS VLab is modular, it can be easily complemented with additional modules to cover more topics.

The rest of the paper is organized as follows: Section I-A briefly explains the aims of the modules taught. Section II outlines the structure of the virtual lab exercises developed. Sections III, IV and V describe a selection of these virtual experiments. Section VI gives the results of the student assessment of these virtual experiments. Finally, Section VII draws conclusions.

\section{A. Modules Taught}

The following three elective modules in the M.Sc. in ICT Systems are allocated 30 teaching hours over a 10-13 week term. As the subject matter content of these modules is rather large, covering both fundamental background knowledge and current state-of-the-art technology, virtual experiments and GUI demonstrations can assist in the educational process by helping students assimilate the concepts. 
1) Multimedia Content Management: This course introduces students to the basic properties of multimedia, which is the principal content transmitted over modern communication networks. Among other issues, the main topics discussed are the coding and transmission of multimedia content, along with their principal applications in modern communication networks and personal appliances. The VLab GUIs and exercises created to facilitate the teaching of these concepts are summarized in Sections III and IV.

2) Medical Imaging: This course aims to provide a thorough understanding of the various imaging representations in modern medicine, introducing the basic concepts of medical imaging and image processing tools, and guiding students through the exploitation of modern medical imaging technology in radiotherapy and surgery. The associated VLab experiments, summarized in Section $\mathrm{V}$, cover a variety of image processing tools essential in the medical imaging field, including image equalization, image noise reduction and image registration and fusion.

3) Geographical Information Systems and Remote Sensing: This course provides a basic introduction to Geographical Information Systems (GIS) and to mapping, and their use in sustainable growth applications. Although mainly focused on GIS applications, this module also features two three-hour lectures on remote sensing imaging and applications, such as pansharpening, which are addressed by a specialized VLab exercise.

\section{THE MASTERS ViRTUAL LABORATORY}

The MASTERS VLab consists of 20 GUIs with richinteraction features, developed in Mathworks MATLAB ${ }^{2}$ and SIMULINK ${ }^{3}$. It is divided into four sections or packages: $\mathbf{A}$ to $\mathbf{D}$. The software is accompanied by an extensive tutorial, which can be plugged into MATLABs help environment and which contains user instructions, some theory, and exercise sets. Starting with fundamental concepts and then progressively and hierarchically building the students knowledge, the following topics are addressed:

\section{A. Audio, Image and Video Encoding and Compression}

A1. Entropy Coding

A2. Scalar and Vector Quantization

A3. Transform Coding

A4-5. "Psychoacoustic masking" and the MP3 (MPEG Audio Layer III) audio-coding standard

A6-7. The JPEG and JPEG-2000 compression standards

A8-10. MPEG1 and MPEG2 video coding

A11. MPEG4 video coding

B. Video and Audio Streaming over Noisy Channels

B1-2. Digital Video Broadcasting - Terrestrial (DVB-T)

B3. Internet Low Bit-rate audio Codec (iLBC)

C. Processing, Registration and Fusion of Medical Images

C1. Image Equalization

C2. Image Noise Reduction

C3-5. Image Registration and Fusion

\footnotetext{
${ }^{2}$ http://www.mathworks.com/products/matlab/

${ }^{3} \mathrm{http}: / /$ www.mathworks.com/products/simulink/
}

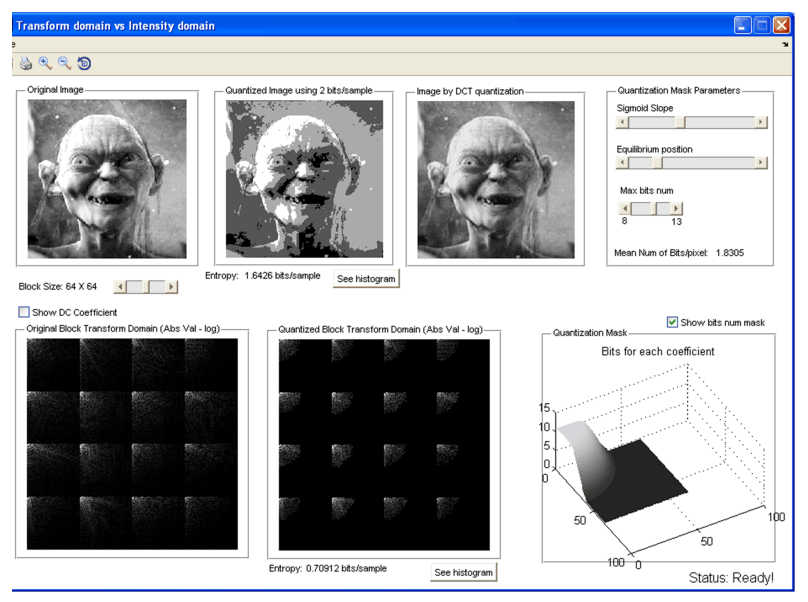

Fig. 1. The Transform Coding GUI.

\section{Fusion of Remote-Sensing Images}

D1. Pansharpening.

The MATLAB programming environment was selected for the MASTERS software development, since MATLAB is highly optimized for vectorized code and therefore suitable for DSP algorithms, it includes rich and well-validated toolboxes for signal and image processing and it provides excellent cross-platform portability. Appropriate wrappers can be developed for importing code written in other languages. Therefore a small portion of the MASTERS VLab code was written in $\mathrm{C}$ to accelerate performance, and many optimized opensource $\mathrm{C}++$ libraries were also used [5]. Using the MATLAB compiler ${ }^{4}$, stand-alone executable applications can be compiled and distributed to users without a MATLAB installation. Furthermore, Mathworks' SIMULINK was used to develop the simulation modules of package B. A number of publiclyavailable libraries [5] were also employed to complement those available in MATLAB.

A significant criterion while developing the VLabs software platforms was adaptability and extendability, so that additional features can be added easily, according to the tutors needs. The following sections present only a portion of the MASTERS VLab GUIs, due to space limitations. The reader is invited to download and explore the whole MASTERS VLab from [5].

\section{Audio-Image-Video EnCoding ANd Compression}

\section{A. Transform Coding}

The compression problem can be divided into a sequence of three relatively simple steps: a) computation of a linear transformation of the data, designed primarily to produce uncorrelated coefficients, such as the Discrete Cosine Transform (DCT) [6], b) quantization of transform coefficients, and c) entropy coding. This process is called transform coding [7] and its principles apply equally well to audio and images in most state-of-the-art compression schemes. The most important concepts of transform coding can be studied and effectively demonstrated using the graphical interface of Fig. 1. Due to its

\footnotetext{
${ }^{4}$ http://www.mathworks.com/products/distriben/
} 
simplicity and its application in many image and video coding standards, the 2D DCT was selected for this interface.

The basic interfaces operations can be summarized as: a source image file is selected by the user. Then, the 2D DCT transform is applied, with the block size specifiable by the user. The user can observe that for natural images the energy is concentrated in low-frequency coefficients. Next, the transform coefficients are quantized using a quantization matrix designed by the user. The applied "quantization mask" can be modified using a rich set of related controls. The quantized transform coefficients are shown. Additionally, the histogram of the coefficients can be displayed (this is not shown in Fig. 1). The user will observe that the histogram is concentrated around the zero-value, which results in low entropy. The entropy of the quantized coefficients is also presented ( 0.7091 bits/coefficient in the example shown). Then the inverse DCT is applied to the quantized transform coefficients, and the resulting compressed image (without using entropy coding) is displayed. For the example shown in Fig. 1, the result was obtained using 1.8305 bits per coefficient; in order to demonstrate the effectiveness of Transform Coding versus direct quantization of the intensity pixel values, the initial image is quantized using $\lceil 1.8305\rceil=2$ bits/pixel and the resulting image is shown. Uniform quantization applied directly to the initial image produces far inferior results to those obtained by DCT quantization. Furthermore, the entropy of the quantized DCT coefficients is much lower ( 0.7091 vs 1.6426 bits/pixels in the shown example), implying that the use of an entropy-coding algorithm would lead to increased compression ratios.

\section{B. Psychoacoustic Analysis and MP3 Audio Coding}

The main modules of the MP3 (MPEG Audio Layer III) coder are the 32-channel polyphase filterbank, the ModifiedDCT transformation that allows short-time processing of the input waveform, the psychoacoustic analysis and the quantizer and entropy coder [8]. The most important feature of the MP3's lossy compression algorithm is that it reduces the accuracy of certain parts of the audio spectrum beyond most peoples ability to resolve.

Two of the MASTERS VLab GUIs can be used to demonstrate the psychoacoustic analysis and the MP3 encoding procedure. In the psychoacoustics GUI, the student can load a particular audio waveform, select an audio segment to be analysed and then study all the individual steps for the computation of global masking thresholds for each audio frame (global peaks, tonal peaks, non-tonal peaks and absolute threshold). With the second GUI (Fig. 2), the student can study the MP3 encoding process by opening an audio file of choice, setting the target MP3 compression bit rate and applying compression. Each (selected) audio frame is displayed in the time domain for the original and the compressed signal respectively. Additionally, the Fourier transform of the original and compressed frames, and their difference, are plotted. The user can thus identify the frequency components discarded by the encoder. For the example of Fig. 2, it can be seen that, based on psychoacoustic analysis and in order to achieve the wanted bit-rate, the encoder eliminated almost all of the high

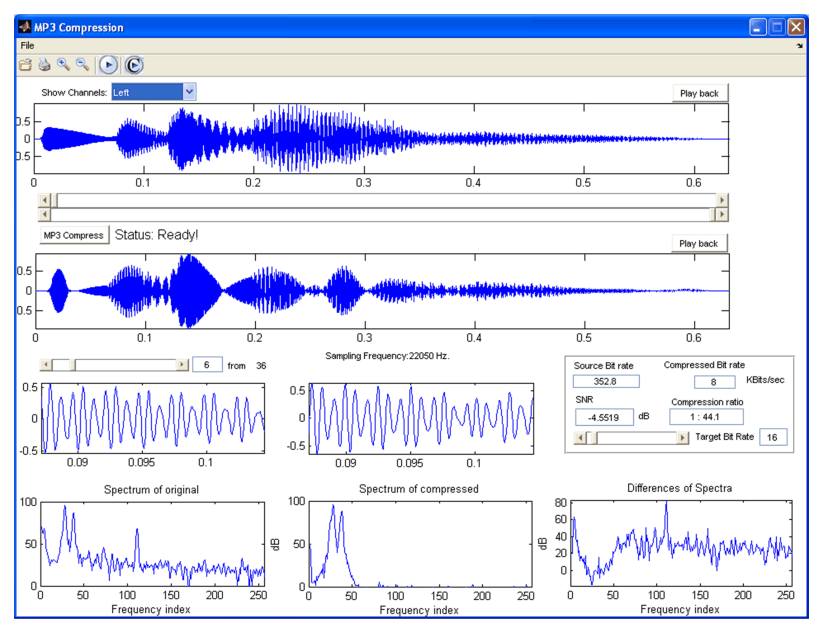

Fig. 2. The MPEG Layer III (MP3) Audio Coding GUI.

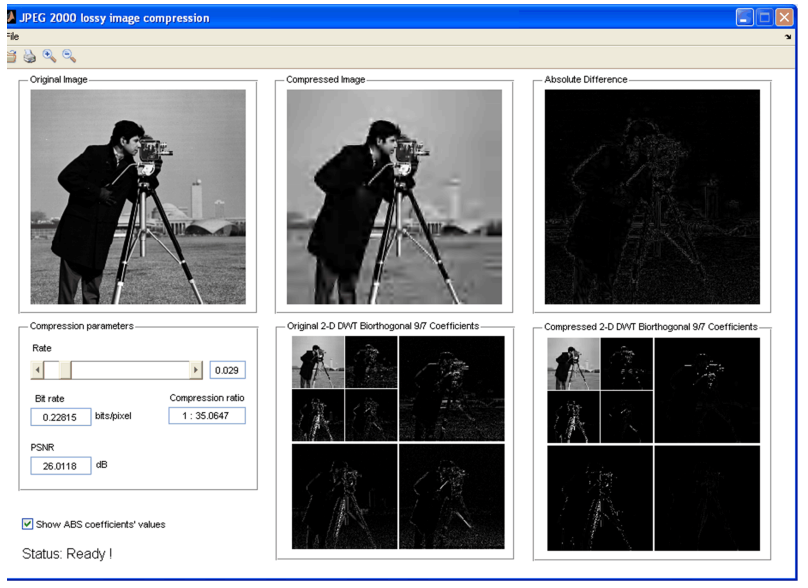

Fig. 3. The JPEG-2000 GUI.

frequency components. Various evaluation metrics are also provided for comparison (SNR, compression ratio, compressed bit rate).

\section{JPEG-2000 Image Coding Standard}

In 2000 the JPEG committee introduced a new method of compressing images, based on the 2D Discrete Wavelet Transform (DWT) [9]. A notable breakthrough was the idea of embedded zero-tree wavelet (EZW) coding, introduced by Shapiro [10]. Improvements and enhancements to the EZW algorithm resulted in modern wavelet encoders [11] with improved performance relative to block transform coders, such as the classic JPEG. Consequently, the JPEG-2000 standard [12] adopted wavelet-based coding as the underlying method. More detailed information on wavelet-based image coding and the JPEG-2000 standard can be found in [11] and [12], respectively.

The MASTERS VLab includes a GUI for studying and demonstrating the effectiveness and the basic principles of operation of JPEG-2000, Fig. 3. This GUI can be used to demonstrate the DWT, the quantization of its coefficients and the concept of zero-wavelet-trees (EZW coding), i.e., the basic elements in JPEG2000, without going into theoretical 
details. The GUI's basic operations can be summarized as follows: the source image is selected; the desired JPEG-2000 compression parameters are specified by the user, with the bit-rate and the corresponding compression-factor being given; finally, the compressed image is displayed in conjunction with the original one. Their difference image is also given, together with the corresponding PSNR (Peak Signal to Noise Ratio). Given that the student has already studied the JPEG standard, this means that he/she can observe the advantages of JPEG2000, and the differences between JPEG-2000 and the classic JPEG, which include the improvement of the compression performance, especially at low bit-rates and reduced visual artifacts: JPEG-2000 produces ringing artifacts near the edges, while classic JPEG produces both ringing artifacts and "block" artifacts, due to its block-based nature. More importantly, the 2D DWT coefficients of the original and the compressed images are shown, illustrating that the DWT coefficients of the compressed image were obtained by quantizing the DWT coefficients of the original image, and showing the wavelet zero-trees, i.e., when a DWT coefficient in a coarse scale is zero (insignificant), the corresponding coefficients in the detail scales are also zero.

\section{MPEG1-2 Video Coding}

The MPEG (Moving Picture Experts Group) coding standard [6], [13] exploits perceptual compression methods to reduce the data-rate. It reduces information in the spatial frequencies of the images for which the human eye has limited perception, and exploits temporal redundancy, i.e., the similarity between consecutive frames. Within the MASTERS VLab framework, three interactive GUIs were developed to demonstrate the main MPEG concepts. The first targets the understanding of the concepts of motion estimation and interframe coding. The second examines the notion of the Group Of Pictures (GOP), i.e., the sequence of I-, P- and B-frames and the step-by-step compression approach in an MPEG coder. The third demonstrates the compression capabilities of an MPEG coder. Due to space limitation, only two of these are described.

1) Motion Estimation and Inter-Frame Coding: Inter-frame coding via motion-compensated prediction is studied with the GUI shown in Fig. 4. This GUI demonstrates the exhaustive block matching algorithm (EBMA) [6] that can be used for motion estimation in a video coder, and how the motion information can be used to code a video frame (target frame) based on a previous frame (anchor frame). The user opens the desired video file and, using a slider control, selects two consecutive frames (anchor and target frames) of the input video to be displayed and processed. The EBMA motion estimation algorithm is applied. With a rich set of controls, the parameters of the algorithm can be specified, including the size of the matching blocks and the search range. Additionally, the user can select between integer-pixel or half-pixel accuracy for motion estimation. The motion estimation results are displayed in real-time, while the user changes the values of the algorithm's parameters. The motion vectors (MVs) obtained are shown. The original anchor frame is motion-compensated using the estimated MVs. The predicted target frame and its

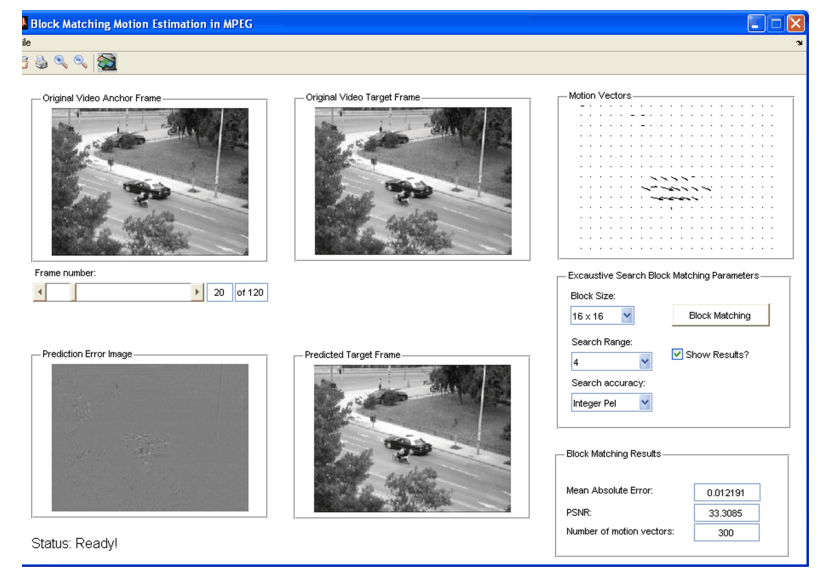

Fig. 4. The block-matching motion estimation GUI

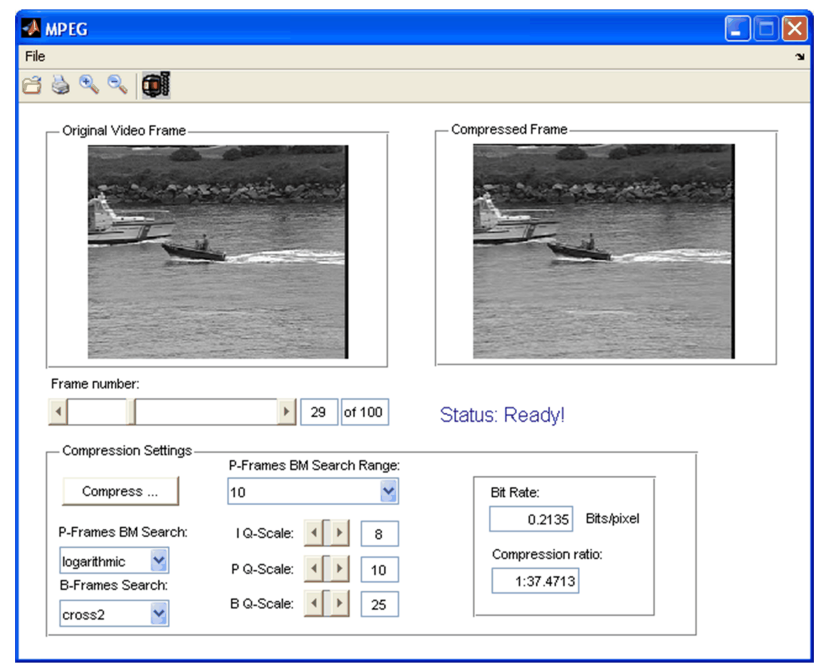

Fig. 5. The MPEG Encoding GUI

difference from the original (i.e., the prediction error image) are displayed, while the corresponding mean absolute error (MAE) and PSNR are also calculated.

2) MPEG Encoder: The GUI shown in Fig. 5 implements a fully-operational MPEG video encoder; this allows the student to study the visual quality of the compressed video vs the compression ratio, by specifying various compression settings [6]. The user selects the video file to be compressed. Various internal compression settings are then specified, including the motion-estimation search algorithm for the $\mathrm{P}$ - and the Bframes, as well as the encoding accuracy for the I-, P- and B-frames (I-, P- and B-scale in Fig. 5)). Applying MPEG compression, the student can view each frame of the compressed video in conjunction with the original. The bit-rate and the compression ratio are also provided. The student can thus study the influence of the internal compression settings on the compression speed, compression efficiency and visual quality.

\section{Audio And Video Streaming}

Digitally-compressed data are considerably vulnerable to transmission errors, due to their use of predictive and variable- 
length (entropy) coding. Therefore, in real-time audio-visual communications, the compressed bit stream has to undergo a channel coding stage using forward error correction (FEC) techniques and interleaving [6], [14], [15]. Digital Video Broadcasting - Terrestrial (DVB-T) transmits MPEG digital video, using coded orthogonal frequency-division multiplexing (OFDM) modulation. The most important modules of the DVB-T transmitter are: the MPEG encoder; an external FEC encoder, using a Reed-Solomon RS $(204,188)$ code; an external convolutional interleaver; an internal FEC encoder, using a punctuated convolutional code; an internal block interleaver, intended to reduce the influence of burst errors; a digital modulation mapper (QPSK, 16-QAM, or 64-QAM); and an OFDM modulator, using 2-K, 4-K, or 8-K OFDM subcarriers.

The GUI illustrated in Fig. 6 is used to simulate DVB-T transmission over noisy channels and video error concealment at the decoder side, using SIMULINK. Specifically, the model simulates: the MPEG video encoder; the channel coding operations, applied by a DVB-T transmitter, i.e., Reed Solomon coding, convolutional encoding, interleaving, or the like; 64QAM digital modulation and 2-K OFDM; transmission over an additive white Gaussian noise (AWGN) channel; demodulation, channel decoding and MPEG decoding; and error concealment of the detected damaged blocks, using motioncompensated temporal prediction [6].

The user can select any input video. The GUI displays in real-time the original input video, the compressed video, the decoded video at the receiver before and after error concealment, and the error video (the difference between the original and the decoded video at the receiver). Furthermore, the user can study the (baseband) OFDM spectrum of the received signal. The user can also change the models parameters during the simulation. The level of AWGN in the transmission channel, specifically the corresponding SNR, can be manually specified in order to observe the change in the bit error rate and the quality of the decoded video (either visually or based on the calculated PSNR) due to transmission errors. The user can specify the number of predicted frames after each I-frame, so as to understand how a damaged block propagates to future frames, due to temporal prediction coding in MPEG. The forward-error (de)coding modules of the (de)coder, as well as the interleaving modules, can be disabled to study their effect and significance. Finally, the user can observe the video quality improvement introduced by the error concealment module of the decoder. Unfortunately, there is no option for the students to capture and process audio or video using the GUI and specialized hardware in real-time. The students can only load previously-recorded audio and video segments. As these (especially video streaming) are rather computationally expensive activities, the speed of the GUI developed depends on the host PC. However, using a Pentium Core 2 at $3 \mathrm{GHz}$, the developed GUI can process audio and video segments of reasonable resolution (e.g. $640 \times 480$ pixels) at near real-time (approximately 10 frames per second).

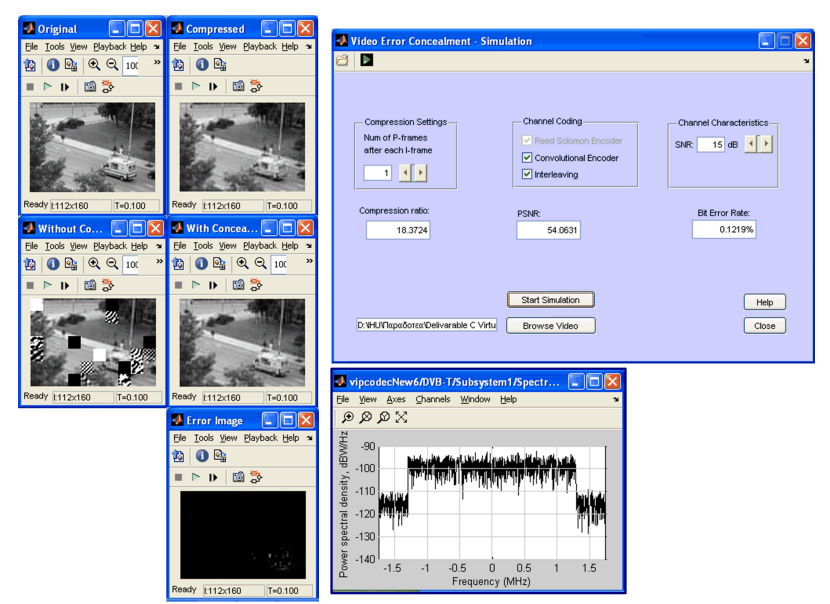

Fig. 6. The DVB-T GUI with error concealment strategies.

\section{Medical Image Processing}

\section{A. Image Denoising}

A great many image denoising methods can be found in the literature, ranging from simple linear or non-linear low-pass filtering to sophisticated non-linear methods, such as transform coefficient shrinkage methods [16]-[18]. The introduction of the wavelet transform (WT) [9] has led to dramatic progress in image denoising over recent decades. The use of non-separable space-frequency transforms, the socalled X-lets (e.g., ridgelets, curvelets, contourlets) [18], [19], in a similar coefficient-shrinkage manner, can lead to noise reduction methods with better edge-preserving characteristics. Recently, a whole new group of adaptive transformation methods using independent component analysis (ICA) was introduced [17]. ICA-based image denoising operates similarly to any transform-based approach, by shrinkage of the transform coefficients.

The GUI illustrated in Fig. 7 demonstrates the various types of noise, the idea of image denoising and various types of denoising algorithms. A user can open any input image, add noise of various types/characteristics to selected regions of interest (ROIs) and finally apply the selected denoising algorithm. The available noise types include additive white Gaussian noise (AWGN), intensity-dependent additive Gaussian noise (AGN), Poisson, salt and pepper noise and multiplicative noise. The appearance of the noise characteristics panel changes according to the noise type selected. Whenever the noise type or its parameters change, the noisy image is automatically updated and the corresponding SNR is displayed. The user can then select the denoising algorithm to apply: simple low-pass filtering; wavelet transform (WT)based denoising; contourlet transform (CoT)-based denoising; and independent component analysis (ICA)-based denoising. The form of the denoising algorithm parameters" panel changes according to the selected denoising algorithm. When the denoising algorithms parameters are specified and the algorithm is applied, the denoised image and the corresponding SNR are displayed. More details on this GUIs operations and its specifiable parameters are available in the MASTERS VLab 


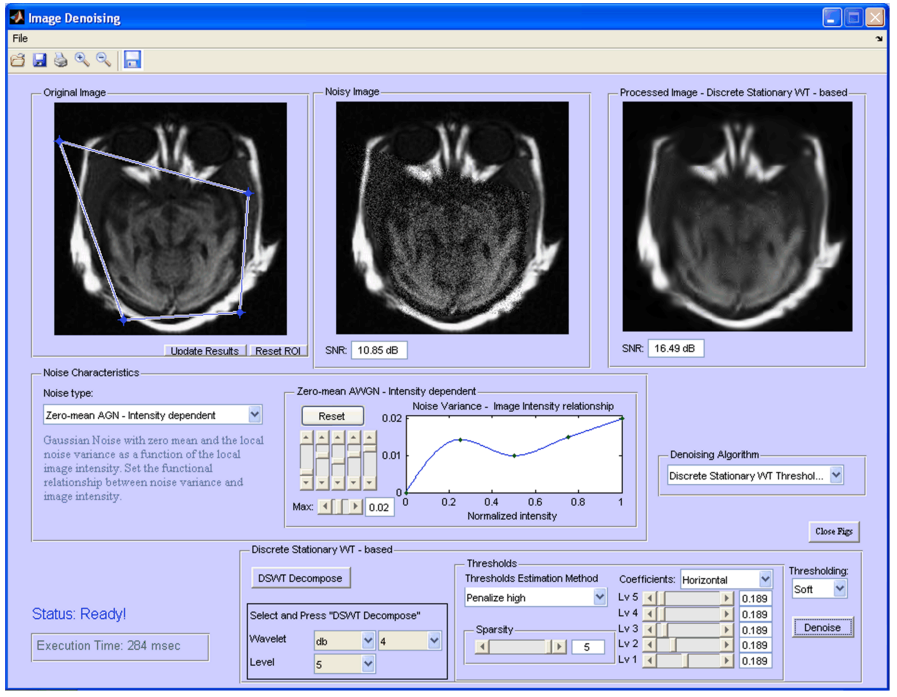

Fig. 7. The Image Denoising GUI

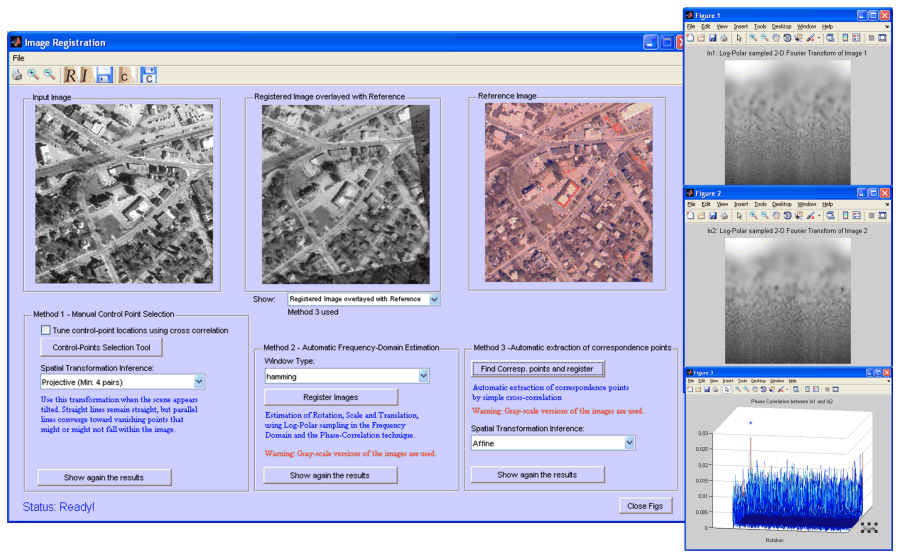

Fig. 8. The Image Registration GUI

tutorial [5].

\section{B. Image Registration}

Image registration, a necessary preprocessing step in computer vision, medical imaging and remote sensing applications, is the process of aligning two or more images of the same object/scene, taken from different viewpoints and/or by different sensors [20]. Modern registration techniques include featurebased [21] and spatial frequency domain [22], [23] methods. The latter methods work for simple transformations, such as translation, rotation and scaling, and are generally faster (due to the existence of fast FFT algorithms) and more robust against illumination changes, noise and/or occlusions.

The GUI illustrated in Fig. 8 helps students understand the notion of image registration by studying three different algorithms: the first based on the manual selection of featurepoint pairs; the second working in the spatial frequency domain; and c) the third based on automatic feature points estimation. The user can open two source image files and apply one of the three available image registration algorithms to align the input images.
1) Manual Registration Method: MATLABs ControlPoints-Selection Tool" is used for manual feature-point selection. The tool displays the two images to be aligned and lets the user select pairs of feature-points. When the tool is closed, the program infers the transformation of the coordinates between the two images. The GUI lets the user store the defined control points in a file for future use. The software can also automatically tune the selected control points, based on a local cross-correlation approach, around each control point. The model of the spatial transformation (affine, projective, etc.) to be estimated is specifiable by the user.

2) Frequency-Domain Method: The student can study a frequency-domain registration method, which calculates the 2D FFTs of the images and resamples the FT amplitudes in log-polar coordinates. Therefore scaling and rotation in the original image intensity domain is manifested as simple translation in the transform domain. This translation (and consequently the scaling and rotation in the original domain) is estimated using the phase-correlation (PC) methodology [22]. In order to suppress the finite image dimension effects in the correlation method, a 2D window can be applied before calculating the PC function. The $2 \mathrm{D}$ window used is specifiable by the user. Three figures with intermediate results appear, Fig. 8, displaying the 2D FFTs of the input images in log-polar coordinates, as well as the corresponding PC function. The position of the peak in the PC function gives the scaling factor and the rotation between the input images.

3) Simple Automatic Feature-Point-Based Method: Finally, the user can study an image registration method that automatically finds feature-point correspondences between the input images. As feature points are considered to be local regions of the images with high variance, point correspondences are found based on a simple local correlation approach. The automatically-extracted feature points and correspondences are presented to the user, and the program infers the coordinates transformation between the two images. The spatial transformation model used is specifiable by the user.

\section{STUdEnT Assessment}

The MASTERS VLab was used by students in the ICT M.Sc. program at IHU, in an elective postgraduate course which was has been offered for two years, 2011 and 2012. The total intake was 31 students, of whom 13 took the elective Multimedia Content Management course module. The other two modules described in Section I-A, did not attract enough students to be offered but are presented for the benefit of instructors who might want to use them. Some of the students who took the Multimedia Content Management module had a computer science/electrical engineering undergraduate background, Fig. 9, but some had only a very brief or no introduction at all to the basics of signal and image processing in their first degree (especially Physics and Mathematics graduates). The course consisted of ten three-hour theory-exercise lectures, taught by a visiting faculty member of staff. The virtual lab was used during lectures to demonstrate some of the concepts taught. The students could spend a total of ten hours (five two-hour sessions) over the semester using the virtual 


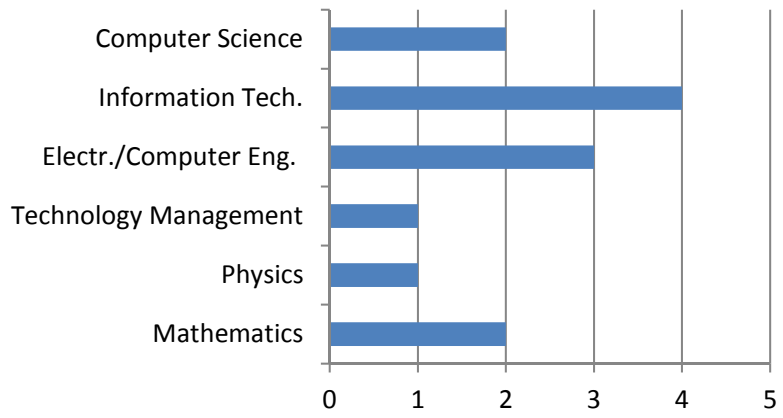

Fig. 9. Undergraduate degrees of the M.Sc.s students taking the Multimedia Content Management module.

labs in the campus computer laboratory, under the supervision of a lab tutor, to complete their assigned coursework. They were also encouraged to download and use the virtual lab at home to complete their assignment or to study for their exams. After the course, all the students were asked to complete an anonymous questionnaire, responding to questions on a fivepoint scale (1-strongly disagree, 2-disagree, 3-neutral, 4-agree, 5 -strongly agree). The results are summarized in Table I.

The goal was to find if the VLab offered learning benefits to such a diverse student audience, especially to those with very limited previous contact with signal processing. The evaluation indicated the MASTERS VLab enhanced students understanding of the course material, with a rating of 4.4/5; this is an important finding, as this was the main motivation behind developing the virtual lab. The GUIs were found to be user friendly and easy to navigate with a rating of 4.6/5. In general, the virtual lab seemed to stimulate students interest in multimedia; together with the course material these given a $4.5 / 5$, which is quite encouraging. The exercise sets that accompanied each GUI had the lowest rating of 3.9/5, which implies that they need improvement. The student feedback stated that the exercise sets were rather elementary compared to the level of understanding required for the course. These exercise sets will be enriched with a variety of problem sets, available in many DSP and image processing textbooks, such as [1], [8]. More specifically, the course tutors are thinking of enriching and dividing the exercise sets into three levels of difficulty (beginner, intermediate, expert) for the next academic year to accommodate multiple levels of expertise from undergraduate and non-expert audiences (introductory) to postgraduate and Ph.D. audiences (expert).

\section{CONCLUSIONS}

The numerous applications of digital signal and image processing in diverse fields makes this an integral part of many undergraduate and postgraduate curricula worldwide. However, the cumbersome theory behind DSP applications makes them difficult to teach, especially to non-expert audiences. Advances in programming platforms, however, and the nature of the concepts behind DSP applications, enables the demonstration of these concepts through interactive GUIs, aiding in their understanding. This paper presented the MASTERS VLab, a package of several GUIs for demonstrations and practice, successfully used at the School of Science and Technology IHU. It addresses several topics in: audio, image and video compression; video and audio streaming over noisy channels; processing, registration and fusion of medical images; and fusion of remote-sensing images. It also assists tutors with inclass demonstrations and provides an interactive opportunity for postgraduate students to experiment, revise and enhance their understanding of the concepts taught. The MASTERS VLab is freely available online for educational and research purposes [5]. The authors hope that this paper will attract the attention of instructors and postgraduate students worldwide in the general area of signal, image and video processing.

\section{ACKNOWLEDGMENTS}

The authors would like to thank Prof. Ioannis Vlahavas, Dean of School of Science and Technology, IHU, for his close supervision of the Virtual Lab project. The authors would also like to thank the students at IHU for evaluating the MASTERS Virtual Lab.

\section{REFERENCES}

[1] S. Mitra, Digital Signal Processing, McGraw-Hill, 2011.

[2] J.H. McClellan, R.W. Schafer, and M.A. Yoder, "A changing role for DSP education," IEEE Signal Processing Magazine, vol. 15, pp. 16-18, 1998.

[3] U. Rajashekar, G.C. Panayi, F.P. Baumgartner, and A.C. Bovik, "The SIVA demonstration gallery for Signal, Image, and Video Processing Education," IEEE Signal Processing Magazine, vol. 45, pp. 16-18, 2002.

[4] S. Shearman, S. Hong, and E. Perez, "To improve Signal Processing concept demonstration software, consider the interactive experience," in 13th IEEE Digital Signal Processing Workshop and 5th IEEE Signal Processing Education Workshop, 2009. DSP/SPE 2009., 2009.

[5] Medical IHU Virtual Labs Multimedia in Telecommunications and Remote Sensing Applications, "http://vlabs.ihu.edu.gr/index.php?id=30,"

[6] Y. Wang, J. Ostermann, and Y. Zhang, Video Processing and Communications, New Jersey: Prentice Hall, 2002.

[7] V.K. Goyal, "Theoretical foundations of Transform Coding," Signal Processing Magazine, vol. 18(5), pp. 9-21, Sep. 2001.

[8] A. Spanias, T. Painter, and V. Atti, Audio signal processing and coding, Wiley-Interscience publication, 2007.

[9] M. Vetterli and J. Kovacevic, Wavelets and Subband Coding, Englewood Cliffs, NJ: Prentice-Hall, 1995.

[10] J. Shapiro, "Embedded image coding using zerotrees of wavelet coefficients," IEEE Trans. Signal Processing, vol. 41, pp. 3445-3462, Dec. 1993.

[11] B.E. Usevitch, "A tutorial on modern lossy wavelet image compression: Foundations of JPEG 2000," Signal Processing Magazine, vol. 18(5), pp. 22-35, Sep. 2001.

[12] A. Skodras, C. Christopoulos, and T. Ebrahim, "The JPEG-2000 still image compression standard," IEEE Signal Processing Magazine, vol. 18(5), pp. 36-58, Sep. 2001.

[13] K.R. Rao and J.J. Hwang, Techniques and Standards for Image, Video and Audio coding, New Jersey: Prentice Hall, 1996.

[14] S.G. Wilson, Digital Modulation and Coding, Englewood Cliffs, New Jersey: Prentice-Hall, 1996.

[15] B. Vucetic and J. Yuan, Turbo codes: principles and applications, Springer Verlag, 2000.

[16] D.L. Donoho, "De-noising by soft-thresholding," IEEE Trans. on Inf. Theory, vol. 41(3), pp. 613-627, 1995.

[17] A. Hyvarinen, P.O. Hoyer, and E. Oja, Image denoising by sparse code shrinkage, Intelligent Signal Processing, IEEE Press, 2001.

[18] J.L. Starck, E.J. Candes, and D.L. Donoho, "The curvelet transform for image denoising," IEEE Trans. on Image Processing, vol. 11(6), pp. 670-684, June 2002.

[19] M.N. Do and M. Vetterli, "The Contourlet Transform: an efficient directional multiresolution image representation," IEEE Trans. on Image Processing, vol. 14, pp. 2091-2106, 2005. 
TABLE I

QUESTIONNAIRE AND RESULTS

\begin{tabular}{|c|c|c|c|c|c|c|}
\hline Question & 1 & 2 & 3 & 4 & 5 & AVG \\
\hline 1. Is the course challenging and interesting? & 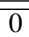 & 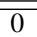 & 1 & 4 & 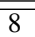 & 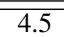 \\
\hline 2. Were the course's theoretical aspects clearly demonstrated by the MASTERS virtual lab ? & 0 & 0 & 1 & 10 & 2 & 4.1 \\
\hline 3. Has the MASTERS virtual lab enhanced your understanding of the taught subjects ? & 0 & 0 & 1 & 6 & 6 & 4.4 \\
\hline 4. Have the proposed exercise sets after each experiment assisted in discovering new concepts of the subjects taught? & 0 & 1 & 4 & 3 & 5 & 3.9 \\
\hline 5. Were the Virtual Labs GUIs user-friendly and easy to follow ? & 0 & 0 & 0 & 5 & 8 & 4.6 \\
\hline 6. Did the Virtual Lab exercises increased your interest in the field of multimedia? & 0 & 0 & 1 & 7 & 5 & 4.3 \\
\hline 7. What is your overall evaluation for the course? & 0 & 0 & 0 & 7 & 6 & 4.5 \\
\hline
\end{tabular}

[20] B. Zitova and J. Flusser, "Image registration methods: a survey," Image and Vision Computing, vol. 21, pp. 9771000, 2003.

[21] D. G. Lowe, "Distinctive image features from scale-invariant keypoints," International Journal of Computer Vision, vol. 60(2), pp. 91-110, 2004.

[22] B.S. Reddy and B.N. Chatterji, "An FFT-based technique for translation rotation and scale-invariant image registration," IEEE Trans. Image Process., vol. 5, pp. 12661271, 1996.

[23] D.S. Alexiadis and G.D. Sergiadis, "Estimation of linear parametric distortions and motions in the frequency domain," in XII MEDICON 2010, Chalkidiki, Greece, 2010.

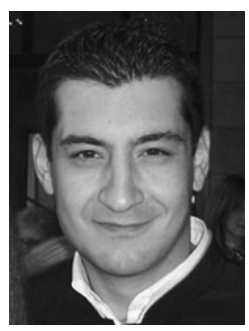

Dimitrios S. Alexiadis (S03) was born in Kozani, Greece, in 1978. He received the Diploma degree and the Ph.D. degree in Electrical and Computer Engineering from the Aristotle University of Thessaloniki (AUTh), Thessaloniki, Greece, in 2002 and 2009 , respectively. Since 2002 , he has been involved in several research projects and has been a Research and Teaching Assistant with the Telecommunications Laboratory, Department of Electrical and Computer Engineering. During 2010, he was a Research Assistant with the School of Science and Technology, International Hellenic University (IHU), Thessaloniki. Since March 2010, he has been a Full-Time Adjunct Lecturer with the Department of Electronics, Technical Education Institute, Thessaloniki. Since January 2011, he has been a Postdoctoral Research Fellow at the Informatics and Telematics Institute, CERTH, Greece. His research interests include still- and movingimage processing, motion estimation; stereo television (TV), high-definition TV, and 3-D TV; detection, estimation, and classification algorithms; and medical image and biomedical signal processing. Dr. Alexiadis is a member of the Technical Chamber of Greece.

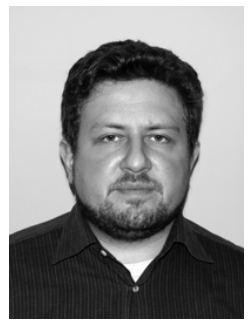

Nikolaos Mitianoudis (S98 - M04 - SM11) received the diploma in Electronic and Computer Engineering from the Aristotle University of Thessaloniki, Greece in 1998. He received the M.Sc. in Communications and Signal Processing from Imperial College London, UK in 2000 and the Ph.D. in Audio Source Separation using Independent Component Analysis from Queen Mary, University of London, UK in 2004. Between 2003 and 2009, he was a Research Associate at Imperial College London, UK, working on the Data Information Fusion-Defense Technology Centre project Applied Multi-Dimensional Fusion, sponsored by General Dynamics UK and QinetiQ. Between 2009 and 2010, he was an Academic Assistant at the International Hellenic University. Since 2010, he has been a Lecturer in Image Processing at the Democritus University of Thrace, Greece. His research interests include independent component analysis, image fusion, computer vision and blind source separation/extraction. 